\title{
BMJ Open Standardised method for reporting exercise programmes: protocol for a modified Delphi study
}

\author{
Susan C Slade, ${ }^{1,2}$ Clermont E Dionne, ${ }^{3}$ Martin Underwood, ${ }^{4}$ \\ Rachelle Buchbinder ${ }^{1,2}$
}

To cite: Slade SC,

Dionne CE, Underwood M, et al. Standardised method for reporting exercise programmes: protocol for a modified Delphi study. BMJ Open 2014;4:e006682. doi:10.1136/bmjopen-2014006682

\section{- Prepublication history for this paper is available online. To view these files please visit the journal online (http://dx.doi.org/10.1136/ bmjopen-2014-006682).}

Received 19 September 2014 Accepted 10 October 2014

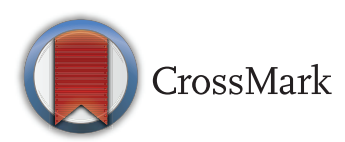

${ }^{1}$ Department of Epidemiology and Preventive Medicine, School of Public Health \& Preventive Medicine, Monash University, Melbourne, Victoria, Australia

${ }^{2}$ Monash Department of Clinical Epidemiology, Cabrini Hospital, Melbourne, Victoria, Australia

${ }^{3}$ Warwick Clinical Trials Unit, Division of Health Sciences, Warwick Medical School, The University of Warwick, Coventry, UK

${ }^{4}$ Department of

Rehabilitation, Faculty of

Medicine, Laval University,

Québec, Canada

Correspondence to Dr Susan C Slade; susan. slade@med.monash.edu

\section{ABSTRACT}

Introduction: Exercise is integral to health across the lifespan and important for people with chronic health conditions. A systematic review of exercise trials for chronic conditions reported suboptimal descriptions of the evaluated interventions and concluded that this hinders interpretation and replication. The aim of this project is to develop a standardised method for reporting essential exercise programme details being evaluated in clinical trials.

Methods and analysis: A modified Delphi technique will be used to gain consensus among international exercise experts. We will use three sequential rounds of anonymous online questionnaires to refine a standardised checklist. A draft checklist of potentially relevant items was developed based on the results of a systematic review of exercise systematic reviews. An international panel of experts was identified by exercise systematic review authorship, established international profile in exercise research and practice and by peer referral. In round 1 , the international panel of experts will be asked to rate the importance of each draft item and provide additional suggestions for revisions or new items. Consensus will be considered reached if at least $70 \%$ of the panel strongly agree/disagree that an item should be included or excluded. Where agreement is not reached or there are suggestions for altered or new items, these will be taken to round 2 together with an aggregated summary of round 1 responses. Following the second round, a ranking of item importance will be made to rationalise the number of items. The final template will be distributed to panel members for approval.

Ethics and dissemination: Ethics approval was received from The Cabrini Institute Ethics Committee, Melbourne, Australia (HREC 02-07-04-14). We plan to use a stepwise process to develop and refine a standardised and internationally agreed template for explicit reporting of exercise programmes. The template will be generalisable across all types of exercise interventions. The findings will be disseminated through peer-reviewed publications and conference presentations.

\section{INTRODUCTION}

Non-communicable diseases, particularly chronic musculoskeletal conditions, are an emerging global issue that must be addressed

\section{Strengths and limitations of this study}

- This study will develop a reporting template that facilitates structured reports of exercise interventions. Poor reporting of exercise programme components has been identified and this limits replication in research and implementation in clinical practice.

- Conducting the study online will facilitate participants' responses (eg, anonymity, accessibility) and the dissemination of information from previous rounds.

- The views of Delphi panellists may differ from those experts who decline participation, and may not fully represent experts in the field of interest. To minimise this limitation, a comprehensive recruitment process involving a systematic review and snowball technique will be used, to ensure a representative range of international researchers and clinicians involved in exercise are invited to participate in the survey.

in the coming decade. ${ }^{12}$ Physical activity and structured regular exercise are integral components of health promotion and key ingredients for health across the lifespan, and are of particular importance for people with chronic conditions. Systematic reviews, ${ }^{3-5}$ clinical practice guidelines ${ }^{6-12}$ and position stands ${ }^{13-15}$ recommend exercise and advice to stay active for the management of many chronic conditions.

Exercise includes activities that vary in type, frequency, intensity, mode and environmental requirements and can vary with respect to type of muscle contraction, load, speed and range of movement, number of repetitions and sets, order of exercises and rest times. It is used to enhance strength, endurance, flexibility, function, and/or achieve specific skill acquisition. These features combine to render 'exercise' an umbrella term for quite different interventions. ${ }^{16-23}$ These may be applied individually or more often applied as a complex intervention comprising two to more different types of exercise interventions. 
A systematic review of 73 systematic reviews of exercise for chronic conditions reported that most reviews were unable to adequately describe the exercise programmes of their included trials because the required information was either not reported or when it was reported, the descriptions varied widely between studies and there was a lack of explicit detail. ${ }^{24}$ For example, the following important domains were not consistently reported: type of exercise, frequency, duration, repetitions, sets, intensity, progression rules, supervision, individual or group and/or adverse events. This is in keeping with the generally poor quality of descriptions of complex interventions in publications more generally. ${ }^{25}$ The lack of a complete description of an intervention limits our ability to draw firm conclusions about its true effects, particularly when conflicting outcomes are reported. In addition it is not possible to replicate it for the purposes of repeating the study, and, if found to be effective, it cannot be reliably implemented.

Evaluation and implementation of physical activity and exercise research would be greatly facilitated if exercise programmes and their components were comprehensively reported in clinical trials in a standardised way. ${ }^{24}$ Based on key exercise descriptors extracted from the included reviews in their systematic review of exercise reviews, ${ }^{24}$ and items recommended in the American College of Sports Medicine (ACSM) models for exercise prescription, Slade and Keating (2012) developed an exercise programme reporting grid that may fulfil this role. The proposed grid includes 43 items that provide explicit data about, for example, whether exercises are generic or individualised, the type of exercise equipment used, the exercise starting position and the programme starting point, the degree of load or resistance, rules for exercise progression, motivation and adherence strategies, and exercise duration, repetitions, sets and sequence. ${ }^{24}$ While comprehensive, this may not be a practical way of summarising the core components of any exercise intervention.

There is a need to develop specific guidance on how exercise interventions should be reported in clinical trials. An internationally endorsed template, and operational manual, for reporting of physical activity and exercise therapy would be a valuable addition to the Consolidated Standards of Reporting Trials (CONSORT) Statement for reporting trials of non-pharmacological treatment, ${ }^{26}$ and could build on work that has produced the recent Template for Intervention Description and Replication (TIDieR), for complex interventions. ${ }^{25}$ Involvement of international experts in developing the template through an international Delphi consensus project is likely to accelerate its uptake.

\section{AIMS}

Using the proposed exercise programme reporting grid by Slade and Keating as a starting point, we will develop and evaluate a standardised method for reporting exercise programmes for chronic musculoskeletal conditions in clinical trials using an international expert consensus Delphi-based survey technique. We will follow the 18-step checklist recommended by Moher $e t a l^{27}$ for the EQUATOR Network, for developing a health research reporting guideline.

Our primary aim is to develop a standardised method for reporting essential details of exercise programmes being evaluated in clinical trials using a modified Delphi technique to reach international consensus.

\section{METHODS AND DESIGN}

The Delphi method is a practical and structured method of obtaining opinions on a given question from a range of experts and is usually used to gain consensus among a group of experts or informed respondents that constitute the Delphi panel. The respondents take part anonymously in sequential questionnaires that constitute different rounds and each round is refined based on feedback from the previous version. After each round, the group responses are fed back to the panellists who can reconsider their views based on this report of the group views. The Delphi method avoids situations in which the group is dominated by the views of a few. ${ }^{28} 29$

We will develop a set of questions, based around the exercise grid items, to send to the experts; the responses will then be collated and distributed back to the participants for further rounds of questions. Rounds will continue until consensus is achieved. Consensus is defined a priori as greater than $70 \%$ agreement on all items with the same ranking. This is aligned with the recommended quality indicators for a Delphi study: reproducible participant criteria, stated number of rounds, clear criteria for excluding/dropping items and other stopping criteria. ${ }^{30}$

\section{Steering Committee}

An international Steering Committee (SCS, CED, MU, RB) was formed to develop and conduct this project and consisted of representation from various disciplines (epidemiology, rheumatology, physical therapy and general medical practice), geographical areas (Australia, UK and North America) and research expertise (qualitative, quantitative and Delphi methods). Research methods were established in face-to-face meetings and email communications. Agreement was reached regarding inclusion/exclusion criteria; a conceptual framework of domains; translation of the original template items into an online survey format; reviews of survey draft iterations until final consensus of item inclusion, question structure and analysis processes. Reference was made to previous and published Delphi studies. ${ }^{31-33}$ There were five drafts reviewed by the Steering Committee, and a further two drafts were developed after a pilot test with four colleagues provided a further set of comments and suggestions.

\section{Generation of the item list}

The items identified from the systematic review ${ }^{24}$ were examined and translated into survey-format questions 
within the following constructs (component parts of theories) and domains (sets of similar theoretical constructs): setting/nature (environmental context/resources, skills// competence/knowledge), process (programme design/ characteristics, programme intention/implementation). These constructs and domains were informed by the Theoretical Domains Framework and implementation research. $^{34} 35$

\section{Selection of international experts (participants and recruitment)}

'Experts' were defined as individuals involved in the conception, design, conduct, teaching or analysis of exercise interventions. We used a purposive and snowball sampling strategy, and because the Delphi group size depends more on group dynamics in reaching consensus among experts than on statistical power, we aimed for the recommended minimum sample size of $20 .{ }^{36}$ To identify experts in the field of exercise research and practice, we reviewed the authorship of published systematic reviews of exercise and physical activity for any condition (including reviews from the Slade and Keating publication); identified established national and international profiles in exercise research and practice; and asked for peer recommendations. To form a representative international expert panel, we sought to include a wide range of professions and research/practice discipline and backgrounds, countries and opinions and any experts who declined participation were asked to suggest a colleague, with similar background, to replace them.

\section{Ethics}

Potential participants will be informed that by responding to the questionnaire, they will be deemed to have consented to participate in the study and have their de-identified responses included in any analyses. All data will be kept on a computer which is password encrypted, in a locked office, in accordance with standard guidelines. Only the researchers will have access to data which will be destroyed after 5 years in accordance with standard guidelines.

\section{Delphi procedure (data collection and data analysis)}

We will use SurveyMonkey software to develop the online survey and invite potential participants via email. The email will include an explanatory statement about the project, the proposed exercise grid and an offer of co-authorship for participants who complete all Delphi rounds of the process. Attrition bias can lead to overestimation of the degree of consensus in the final results. Strategies to prevent attrition bias include only inviting people who respond to a pre-Delphi invitations to participate in the first round or to list, in the publication, only those participants who either completed the entire Delphi process or agree to the final consensus. ${ }^{36}$

Each survey round will be online for up to 4 weeks and reminder emails will be sent approximately every 7 days after the initial invitation. In the first round, participants will be asked to rank the importance of items in the grid by rating each item on an 11-point Likert rating scale $(0-10)$ from 0 : strongly disagree to 10 : strongly agree. They will also be asked to provide recommendations regarding any additions and/or deletions to the list of proposed items and for any other comments/suggestions. Each survey will take 20-40 min to complete, with the facility to complete it over several sessions and to allow participants to review their answers before final submission of their responses.

Only those who complete round 1 will be invited to participate in round 2. De-identified results comprising overall scores for each item (analysed in a number of ways, eg, percentage, mean, median, SD, range and proportions for the quantitative data and thematic analysis for the qualitative data) and narrative summary of findings, comments and suggestions will be sent to each panel member after the first round. In a second round, all ambiguous items or proposals driven by comments of the first round and concerning exclusion, aggregation or retention of items, together with any new potential items identified from the first round, will be included in the second survey. For example, if in round 1 several comments were made about the breadth of a question(s) covering other more specific questions, a proposal will be made to erase the perceived duplicate(s) or combine items. Items that do not reach a consensual mean score of at least 7 out of 10 will be excluded from further consideration.

Following the second round, a ranking of item importance will be made to rationalise the number of items and model this according to the CONSORT Statement and TIDieR Checklist for consistency. ${ }^{25}{ }^{27}$ Synthesis of comments and further additions and deletions will be made until there is final majority agreement. It is anticipated that three rounds will be required. The second round results will also be submitted to an accepted workshop at the Low Back Forum, Brazil 2014 (http://www. lbpforum.com.br) for consensus among low back pain experts regarding item use in exercise programmes for low back pain. To test the acceptability of the approach to our end-users, researchers with an interest in testing the clinical application of exercise programmes, we will seek consensus from these low back pain experts. If the approach is acceptable to this paradigmatic group, we anticipate that it will be acceptable to researchers focused on other chronic non-communicable diseases.

The final exercise grid will be distributed to the panel members for final approval and an operational manual, with decision rules for each item and guidance on how some information may best be presented (eg, in a Table, or as absolute numbers) and elaboration and explanatory document will be developed. Translation and adaptation into languages other than English will also be considered to assist with dissemination.

\section{DISCUSSION}

We plan to use a stepwise process to develop and refine a standardised and internationally agreed on template 
for explicit reporting of exercise programmes in clinical trials. The template should be generalisable across all types of exercise interventions for any conditions. Detailed information about exercise interventions evaluated in clinical trials is necessary for the optimal translation of evidence into clinical and research practice. Use of the template should facilitate provision of explicit details about exercise interventions in clinical trials as a basic standard and is likely to be an important adjunct to the CONSORT and TIDieR templates.

\section{DISSEMINATION PLAN}

The findings of this study will be disseminated through peer-reviewed publications, conference presentations, registration of the guideline with the EQUATOR Network and guideline publication.

\section{DELPHI STUDY STATUS}

The first round of the Delphi study has been completed, analysed and reviewed by the Steering Committee and feedback has been sent to the participants. The second round has been completed and undergoing analysis. The Low Back Pain Forum workshop has been presented and data are being collated and analysed. The third round will be finalised and distributed within 1 month open and followed by a consensus panel. A paper reporting the results of the Delphi study will be submitted for publication in 2015 and conference presentations are planned. Data collection started in June 2014 and anticipated to be completed by December 2014.

Contributors SCS is the principle and coordinating investigator. SCS and RB are responsible for the design of the study and the study protocol. MU and CED have contributed to the design of the study and the content of the study protocol with important intellectual revisions. SCS is responsible for drafting the protocol manuscript. All authors have read and approved the final protocol manuscript.

Funding This research project is supported financially by the Arthritis Australia National Research Program (Philip Benjamin Grant) Number: 2014GIA03.

Competing interests None.

Ethics approval Cabrini Institute Ethics Committee (HREC 02-07-04-14).

Provenance and peer review Not commissioned; internally peer reviewed.

Data sharing statement The authors will share questionnaire items for all survey rounds and these are available from the first author.

Open Access This is an Open Access article distributed in accordance with the Creative Commons Attribution Non Commercial (CC BY-NC 4.0) license, which permits others to distribute, remix, adapt, build upon this work noncommercially, and license their derivative works on different terms, provided the original work is properly cited and the use is non-commercial. See: http:// creativecommons.org/licenses/by-nc/4.0/

\section{REFERENCES}

1. Murray CJ, Vos T, Lozano R, et al. Disability-adjusted life years (DALYs) for 291 diseases and injuries in 21 regions, 1990-2010: a systematic analysis for the Global Burden of Disease Study 2010. Lancet 2012;380:2197-23.

2. Vos $T$, Flaxman $A D$, Naghavi $M$, et al. Years lived with disability (YLDs) for 1160 sequelae of 289 diseases and injuries 1990-2010: a systematic analysis for the Global Burden of Disease Study 2010. Lancet 2012;30:2163-96.

3. Smidt N, de Vet HCW, Bouter LM, et al. Effectiveness of exercise therapy: a best-evidence summary of systematic reviews. Aust $J$ Physiother 2005;51:71-85.

4. Taylor NF, Dodd KJ, Shields N, et al. Therapeutic exercise in physiotherapy practice is beneficial: a summary of systematic reviews 2002-2005. Aust J Physiother 2007;53:7-16.

5. Kujala UM. Evidence on the effects of exercise therapy in the treatment of chronic disease. Br J Sports Med 2009;43:550-8.

6. NICE (National Institute for Health and Clinical Excellence) Clinical Guidelines. http://www.nice.org.uk/;guidance.nice.org.uk/topic

7. National Health and Medical Research Council (Australia). http:// www.nhmrc.gov.au/_files_nhmrc/publications/attachments/ac2.pdf

8. Philadelphia Panel. Philadelphia Panel evidence-based clinical practice guidelines on selected rehabilitation interventions for shoulder pain. Phys Ther 2001;81:1719-30.

9. Koes BW, van Tulder M, Lin CW, et al. An updated overview of clinical guidelines for the management of non-specific low back pain in primary care. Eur Spine J 2010;19:2075-94.

10. Savigny $P$, Kuntze $S$, Watson $P$, et al. Low back pain: early management of persistent non-specific low back pain. London: National Collaborating Centre for Primary Care and Royal College of Surgeons, 2009

11. McAlindon TE, Bannuru RR, Sullivan MC, et al. OARSI guidelines for the non-surgical management of knee osteoarthritis. Osteoarthritis Cartilage 2014;22:363-88.

12. Billinger SA, Arena R, Bernhardt J, et al. Physical activity and exercise recommendations for stroke survivors: a statement for healthcare professionals from the American Heart Association/ American Stroke Association. Stroke 2014;45:2532-53.

13. Garber CE, Blissmer B, Deschenes MR, et al. American College of Sports Medicine. American College of Sports Medicine position stand. Quantity and quality of exercise for developing and maintaining cardiorespiratory, musculoskeletal, and neuromotor fitness in apparently healthy adults: guidance for prescribing exercise. Med Sci Sports Exerc 2011;43:1334-59.

14. Kraemer WJ, Adams K, Cafarelli E, et al. Progression models in resistance training for healthy adults: American College of Sports Medicine Position Stand. Med Sci Sports Exerc 2002;34:364-80.

15. Durstine JL, Moore G, Painter P, et al. American College of Sports Medicine's exercise management for persons with chronic diseases and disabilities. 3rd edn. American College of Sports Medicine, 2009. ISBN-13: 9780736074339.

16. Deschenes MR, Kraemer WJ. Performance and physiologic adaptations to resistance training. Am J Phys Med Rehabil 2002;81 (11 Suppl):S3-16.

17. Kraemer WJ, Ratamess N, Fry AC, et al. Influence of resistance training volume and periodization on physiological and performance adaptations in collegiate women tennis players. Am J Sports Med 2000;28:626-33.

18. Fees M, Decker T, Snyder-Mackler L, et al. Upper extremity weight-training modifications for the injured athlete: a clinical perspective. Am J Sports Med 1998;26:732-42.

19. Campos GE, Luecke TJ, Wendeln HK, et al. Muscular adaptations in response to three different resistance-training regimens: specificity of repetition maximum training zones. Eur J Appl Physiol 2002;88:50-60.

20. Wernbom M, Augustsson J, Thomee R. The influence of frequency, intensity, volume and mode of strength training on whole muscle cross-sectional area in humans. Sports Med 2007;37:225-64.

21. Munn J, Herbert RD, Hancock MJ, et al. Resistance training for strength: effect of number of sets and contraction speed. Med Sci Sports Exerc 2005;37:1622-6.

22. Peterson MD, Rhea MR, Alvar BA. Applications of the dose-response for muscular strength development: a review of meta-analytic efficacy and reliability for designing training prescription. J Strength Cond Res 2005;19:950-8.

23. Fry AC. The role of resistance exercise intensity on muscle fibre adaptations. Sports Med 2004;34:663-79.

24. Slade SC, Keating JL. Exercise prescription: a case for standardised reporting. Br J Sports Med 2011;46:1110-13.

25. Hoffman TC, Glasziou PP, Milne R, et al. Better reporting of interventions: template for intervention description and replication (TIDieR) checklist and guide. BMJ 2014;348:g1687.

26. Altman DG, Sculz KF, Moher D, et al. The revised CONSORT statement for reporting randomized trials: explanation and elaboration. Ann Intern Med 2001:134:663-94.

27. Moher D, Schulz KF, Simera I, et al. Guidance for developers of health research reporting guidelines. PLOS Med 2010;7:e1000217.

28. Sinha IP, Smyth RL, Williamson PR. Using the Delphi technique to determine which outcomes to measure in clinical trials: 
recommendations for the future based on a systematic review of existing studies. PLoS Med 2011;8:e1000393.

29. Boulkedid R, Abdoul H, Loustau M, et al. Using and reporting the Delphi method for selecting healthcare quality indicators: a systematic review. PLOS ONE 2011;6:e20476.

30. Diamond IR, Grant RC, Feldman BM, et al. Defining consensus: a systematic review recommends methodologic criteria for reporting of Delphi studies. J Clin Epidemiol 2014;67:401-9.

31. Dionne CE, Dunn KM, Croft PR, et al. A consensus approach toward the standardisation of back pain definitions for use in prevalence studies. Spine 2008;33:95-103.

32. Froud R, Eldridge S, Kovacs S, et al. Reporting outcomes of back pain trials: a modified Delphi study. Eur J Spine 2011;15:1068-74.
33. Ward L, Stebbings S, Sherman K, et al. Establishing key components of yoga intevrentions for musculoskeletal conditions: a Delphi study. BMC Complement Altern Med 2014;14:96.

34. Michie S, Johnston M, Abraham C, et al. Making psychological theory useful for implementing evidence-based practice: a consensus approach. Qual Saf Health Care 2005;14:26-33.

35. Huijg J, Gebhardt WA, Dusseldorp E, et al. Measuring determinants of implementation behaviour: psychometric properties of a questionnaire based on the theoretical domains framework. Implement Sci 2014;9:33.

36. Okoli C, Pawlowski SD. The Delphi method as a research tool: an example, design considerations and applications. Inform Manag 2004;42:15-29. 